\title{
Norepinephrine: the next therapeutics frontier for Parkinson's disease
}

\author{
Peter A LeWitt
}

\begin{abstract}
Tissue concentrations of norepinephrine (NE) are markedly decreased in various regions of the Parkinson's disease (PD) brain. As in the substantia nigra pars compacta, neuronal dropout and Lewy bodies are prominent changes affecting the locus coeruleus, which is the source of ascending NErgic projections. Despite the major roles of NE throughout the brain, there has been only minimal exploration of pharmacological intervention with NErgic neurotransmission. Cognitive operations, "freezing" of gait, tremor, dyskinesia, REM sleep regulation, and other aspects of brain function are tied into signaling by NE, and there is also evidence that it may have a role in the neurodegenerative process itself. This article reviews the reported pharmacological experience in PD therapeutics.
\end{abstract}

Keywords: Norepinephrine, Parkinson?'?s disease, neuropharmacology

\section{Review}

Successful treatment of Parkinson's disease (PD) with neurotransmitter replacement has placed dopamine "center stage" for understanding the pathophysiology of this disorder. The seminal research of Arvid Carlsson and other investigators in the 1950s elevated dopamine's role from that of a mere metabolic intermediate to the "star of the show" in PD and other brain disorders. Neglected by the attention given to dopamine, however, was the significance of another important CNS neurotransmitter, norepinephrine (NE). In nerve terminals containing the rate-limiting enzyme dopamine- $\beta$-hydroxylase, NE is formed in the next step in catecholamine synthesis beyond dopamine. Like dopamine, NE is involved in a wide range of cognitive, motor, and autonomic functions of the brain. Beyond its roles as a neurotransmitter, the actions of NE are involved in one or more mechanisms linked to neurodegeneration in the PD brain [1]. There has been only limited pharmacological experience exploring the clinical significance of modulating NE neurotransmission. This review will cover the highlights of this therapeutic research experience.

In PD, NE synthesis is greatly decreased throughout the brain. In several regions, NE content is reduced to less than half of its usual tissue concentration [2]. CSF

Correspondence: palewitt1@hfhs.org

Department of Neurology, Henry Ford Hospital and Wayne State University School of Medicine, Detroit, Michigan USA concentration of dopamine- $\beta$-hydroxylase (the rate limiting enzyme for NE synthesis) is also diminished [3]. The loss of NErgic innervation is attributable to the prominent pathology found in the locus coeruleus (LC) [4]. From these paired brainstem structures, ascending projections arise that are distributed widely to the cerebral cortex and deeper structures [5]. Long before the significance of decreased dopaminergic innervation was understood as a key feature of PD, neuropathologists recognized that changes in the $\mathrm{LC}$ were as extensive as those affecting neurons in the substantia nigra pars compacta (SNpc) [6]. Beyond the dropout of NErgic neurons in the $\mathrm{LC}$, the remaining pigmented neurons tend to be affected with Lewy bodies and Lewy neurites (similar to findings for dopaminergic neurons in the SNpc). Neuronal degeneration in LC precedes by several years the development of similar changes in the SNpc [7]. Although the cause(s) in PD for the progressive and relatively selective attack on both the LC and SNpc remains to be learned, neurons in both brain regions share in common an intracellular accumulation of neuromelanin pigment as well as the enzymatic apparatus for catecholamine synthesis and catabolism. These factors may confer vulnerability for neurodegeneration based on oxidative stress from metabolism of the neurotransmitters or their auto-oxidation [8].

Research into the etiology of PD has also given consideration to other ways that NE might be involved. 
One intriguing possibility comes from its influence on inflammatory mechanisms, which have been suspected to be involved in the common final pathway for the pathogenesis of PD (regardless of initiating cause) [9]. In animal experiments, NE inhibits gene expression leading to pro-inflammatory molecules (especially cytokines) originating in microglia, astroglia, and endothelial cells [1]. Other properties associated with NErgic innervation include the reduction of oxidative stress (by lessening the formation of nitric oxide and other intracellular reactive oxygen species), and lessening of both mitochondrial membrane depolarization and caspase activation [10]. As a result, the presence of NE innervation may protect against neurodegeneration in the $\mathrm{SNpc}$ dopaminergic neurons. Evidence for this possibility comes from experimental lesioning of the LC, which adds to the damage of dopaminergic neurons caused by the neurotoxin 1-methyl-4-phenyl-1,2,3,6-tetrahydropyridine (MPTP) [11-13]. In contrast, enhancing NE synthesis counters the toxicity of MPTP against dopaminesecreting neurons in experimental Parkinsonism [13,14]. Taken together, the experimental evidence suggests that a decline in NE synthesis might be a factor in the neurodegenerative disease process of PD.

As with dopamine receptors, adrenoceptors in the nervous system have a complexity that is conferred by both their localization and by their differing signal transduction properties. The effects of NE are governed by an intermingling of receptors with excitatory and inhibitory pre- and post-synpatic functions. NE acts through both through immediate neurotransmission and also by long-term potentiation (which facilitates synaptic plasticity). It also indirectly enhances glutamate release. The NErgic output from the LC has a number of physiological roles in the brain, including activation of cerebral cortex for functions such as vigilance, wakefulness, and circadian rhythms. NE input plays a role in monitoring of arousal and attentional systems, especially those with inherent novelty. Attention mechanisms are enhanced by the influence of NE in the prefrontal cortex, while short-term memory is facilitated by actions of this neurotransmitter in the hippocampus. Pertinent to neurological deficits in PD are additional roles of LC NErgic innervation with respect to autonomic functions and regulation of the sleep-wake cycle. NE afferents from the LC also influence firing patterns of SNpc neurons and release of striatal dopamine [15].

There has been only limited pharmacological research with CNS NE to achieve symptomatic therapy of PD. In several studies, drugs selectively interacting with $\mathrm{NE}$ have offered insight to roles that NE might play in treating motor, cognitive, and affective features of PD. For example, clinical manifestations of resting, postural, and action tremor in PD can be diminished approximately one-third by the $\beta$-adrenoceptor antagonist nadolol [16]. Another $\beta$-blocker, propranolol, was claimed to reduce levodopa-induced dyskinesias in PD patients (and without worsening Parkinsonism) [17]. Levodopa-induced dyskinesias have also been diminished by other interactions with NE neurotransmission. For example, treatment with the $\alpha 2$-adrenergic receptor antagonists idaxozan [18] and fipemazole [19] reduced involuntary movements without a reduction in the anti-Parkinsonian benefits of levodopa.

Other motor features of PD and its therapeutics have been investigated by means of a drug that can selectively enhance NE production in the brain. The L-threo form of dihydroxyphenylserine (L-DOPS, or droxidopa) is an unnatural amino acid developed for pharmaceutical purposes. Although lacking any pharmacological effects by itself, L-DOPS is capable of direct conversion to NE by L-aromatic amino acid decarboxylase (the same enzyme that converts levodopa to dopamine). L-DOPS has been extensively used to treat orthostatic hypotension by increasing peripheral NE synthesis [20]. If the decarboxylase inhibitors carbidopa or benserazide are co-administered with L-DOPS, then most of this NE precursor is blocked from metabolism outside of the CNS. Like levodopa, L-DOPS crosses the blood-brain barrier by a facilitated amino acid transport mechanism.

In Japan, where the drug has been marketed since 1991, L-DOPS underwent investigation to investigate the role of NE in advanced PD. In a multicenter placebo-controlled study, some patients experiencing freezing of gait and retropulsive imbalance exhibited marked clinical improvements from L-DOPS [21]. Following these studies, there have been no further randomized clinical trials, and so the question of L-DOPS effectiveness in the problems of advanced PD awaits further study. Even when L-DOPS is co-administered with a decarboxylase inhibitor, recent clinical experience indicates that this drug can improve symptomatic features of postural hypotension experienced by PD patients [20].

Beyond its effects on motor aspects of PD, the role of $\mathrm{NE}$ in cognitive functions has been of particular interest for pharmacological interventions. Since the LC provides extensive projections to frontal cortex, several investigators have hypothesized that modulating NErgic neurotransmission might help with the various types of cognitive deficits found in PD. In one small-scale study, administering a selective NErgic $\alpha-1$ agonist was associated with improved performance for attentional deficits in nondemented PD patients [22]. Another investigation found that treatment with the $\alpha-2$ adrenoceptor agonist clonidine (which acts to decrease NE release in the brain) led to improved spatial working performance [23]. The NE re-uptake inhibitor atomoxetine showed a trend for improving several frontal lobe 
Table 1 The unmet needs of Parkinson's disease therapeutics

\begin{tabular}{|c|c|}
\hline - Progressive worsening of all clinical features & - Unpredictable immobility (freezing of gait, prolonged "off" states) \\
\hline - Inadequate tremor control & - Autonomic dysfunction (postural hypotension, constipation, dysphagia) \\
\hline $\begin{array}{l}\text { - Balance impairment (particularly retropulsive } \\
\text { imbalance) }\end{array}$ & - Sleep disturbance (fragmented sleep, rapid-eye-movement behavioral disorder) \\
\hline - Postural disturbance (forward flexion) & - Depression, fatigue, and apathy \\
\hline $\begin{array}{l}\text { - Involuntary movements with dopaminergic therapy } \\
\text { (dyskinesia, dystonia) }\end{array}$ & $\begin{array}{l}\text { - Progressive cognitive decline (including impaired working memory and executive } \\
\text { dysfunction even in the absence of dementia) }\end{array}$ \\
\hline
\end{tabular}

measures of cognitive performance in a small open-label study of non-demented PD patients [24]. Apart from these few clinical investigations, the role of NE in the range of $\mathrm{PD}$-associated cognitive impairments [25] has not been explored.

The increased risk for occurrence of depression affecting PD patients has been associated with neurochemical changes that include the LC projections to limbic structures [26]. As a result, the reduction in NE neurotransmission may offer a pharmacological target. This possibility was explored in a clinical trial testing whether reboxetine, a selective NE re-uptake inhibitor, could offer improvement for a major depressive episode in PD patients. With this drug, the outcome was quite striking, as shown by improvements in Hamilton Depression Scale scores that were comparable to the best that serotonin-selective reuptake inhibitors achieve in treating depression for non-PD populations [27]. During this clinical trial, no changes occurred in ratings of Parkinsonian severity.

The studies described above constitute the sum of investigations in PD with respect to $\mathrm{NE}$ and the various deficits associated with this disorder. Given the importance of NErgic innervation in the brain and its major loss in PD, it would seem that this neglected neurotransmitter deserves greater pharmacological attention. Dopaminergic therapy of PD has greatly changed the options for achieving a lasting control of PD disabilities. Despite this, there are many continuing challenges for the further development of PD therapeutics (Table 1). Some of these unmet needs may be related to the various roles of NE in the brain. The studies described above, however meager, suggest that augmenting or blocking NE neurotransmission has the potential to improve several motor and non-motor problems of PD. Furthermore, NE's possible role at protecting against the progression of PD awaits study. One opportunity to explore the role of NErgic functions in the brain and systemically is with the NE precursor L-DOPS, which offers a selective pharmacological probe for augmenting NE neurotransmission. Using L-DOPS, the author is planning to initiate clinical trials to study the effects of increasing brain NE for treating cognitive impairments and freezing of gait in PD patients.
Competing interests

The authors declare that they have no competing interests.

Received: 6 December 2011 Accepted: 13 January 2012

Published: 13 January 2012

\section{References}

1. Gesi M, Soldani P, Giorgi FS, et al: The role of the locus coeruleus in the development of Parkinson's disease. Neurosci Biobehav Rev 2000, 24:655-668.

2. Agid $Y$, Javoy-Agid F, Ruberg M: Biochemistry of neurotransmitters in Parkinson's disease. In Movement Disorders 2. Edited by: Marsden CD, Fahn S. New York: Butterworths; 1987:166-230.

3. Hurst JH, LeWitt PA, Burns RS, Foster NL, Lovenberg W: CSF dopamine $\beta$ hydroxylase activity in Parkinson's disease. Neurology 1985, 35:565-568.

4. Chan-Palay $V$, Asan A: Alterations in catecholamine neurons of the locus coeruleus in senile dementia of the Alzheimer type and in Parkinson's disease with and without dementia and depression. J Comp Neurol 1989, 287:373-392.

5. Benarroch EE: The locus coeruleus norepinephrine system: functional organization and potential clinical significance. Neurology 2009, 73:1699-1704.

6. Greenfield JG, Bosanquet FD: The brainstem lesions in Parkinsonism. J Neurol Neurosurg Psychiatry 1953, 16:213-226.

7. Del Tredici K, Rüb U, De Vos RA, Bohl JR, Braak H: Where does Parkinson disease pathology begin in the brain? J Neuropathol Exp Neurol 2002, 61:413-426.

8. Götz ME, Künig G, Riederer P, Youdim MB: Oxidative stress: free radical production in neural degeneration. Pharmacol Ther 1994, 63:37-122.

9. Hirsch EC, Hunot S: Neuroinflammation in Parkinson's disease: a target for neuroprotection? Lancet Neurol 2009, 8:382-397.

10. Rommelfanger KS, Weinshenker D: Norepinephrine: the redheaded stepchild of Parkinson's disease. Biochem Pharmacol 2007, 74:177-190.

11. Mavridis M, Colpaert FC, Millan MJ: Differential modulation of (+)amphetamine-induced rotation in unilateral substantia nigra-lesioned rats by alpha- 1 as compared to alpha 2 agonists and antagonists. Brain Res 1991, 562:216-224.

12. Marien M, Briley M, Coplaert F: Noradrenaline exacerbates MPTP-induced striatal dopamine loss in mice. Eur J Pharmacol 1993, 236:487-489.

13. Fornai F, Bassi L, Torracca MT, Scalori V, Corsini GU: Norepinephrine loss exacerbates methamphetamine-induced striatal dopamine depletion in mice. Eur J Pharmacol 1995, 283:99-102.

14. Kilbourn MR, Sherman P, Abbott LC: Reduced MPTP toxicity in striatum of the mutant mouse tottering. Synapse 1998, 30:205-210.

15. Grenhoff J, Nisell M, Ferre S, et al: Noradrenergic modulation of midbrain dopamine cell firing elicited by stimulation of the locus coeruleus in the rat. J Neural Transm Gen Sect 1993, 93:11-25.

16. Foster NL, Newman RP, LeWitt PA, et al: Peripheral beta-adrenergic blockade treatment of Parkinsonian tremor. Ann Neurol 1984, 16:505-508.

17. Carpentier AF, Bonnet AM, Vidhailhet M, Agid Y: Improvement of levodopainduced dyskinesia by propranolol in Parkinson's disease. Neurology 1996, 46:1548-1551.

18. Rascol O, Arnulf I, Peyro-Saint Paul H, et al: Idoxozan, an alpha-2 antagonist, and L-DOPA-induced dyskinesias in patients with Parkinson's disease. Clin Neuropharmacol 2001, 16:708-713.

19. LeWitt PA, Hauser RA, Lu M, et al: Fipamezole in the treatment of dyskinesias in advanced Parkinson disease (FJORD study). Neurology 2011. 
20. Mathias CJ: L-dihydroxyphenylserine (droxidopa) in the treatment of orthostatic hypotension: the European experience. Clin Auton Res 2008, 18(suppl 1):25-29.

21. Narabayashi H, Nakanishi T, Kanazawa I, et al: Clinical effects of L-threo-3,4dihydroxyphenylserine in Parkinson's disease and Parkinson syndrome: results of multi-center open study at 45 institutions. Yakuri To Chiryo [Japan Pharmacol Ther; in Japanese] 1987, 15(suppl 2):411-443.

22. Bédard MA, el Massioui F, Malapani C, et al: Attentional deficits in Parkinson's disease: partial reversibility with naphtoxazine (SDZ NVI-085), a selective noradrenergic alpha 1 agonist. Clin Neuropharm 1998, 21:108-117.

23. Riekkinen $M$, Jäkälä $P$, Kejonen $K$, Riekkinen $P$ Jr: The alpha2 agonist, clonidine, improves spatial working performance in Parkinson's disease. Neuroscience 1999, 92:983-989.

24. Marsh L, Biglan K, Gerstenhaber M, Williams JR: Atomoxetine for the treatment of executive dysfunction in Parkinson's disease: a pilot openlabel study. Mov Disord 2009, 24:277-282.

25. Dubois B, Pillon B: Cognitive deficits in Parkinson's disease. J Neurol 1997, 244:2-8.

26. Remy P, Doder M, Lees A, Turjanski N, Brooks D: Depression in Parkinson's disease: loss of dopamine and noradrenaline innervations in the limbic system. Brain 2005, 128:1314-1322.

27. Pintor $L$, Baillès $E$, Valldeoriola $F$, et al: Response to 4-month treatment with reboxetine in Parkinson's disease patients with a major depressive episode. Gen Hosp Psychiatry 2006, 28:59-64.

doi:10.1186/2047-9158-1-4

Cite this article as: LeWitt: Norepinephrine: the next therapeutics

frontier for Parkinson's disease. Translational Neurodegeneration 2012 1:4.

\section{Submit your next manuscript to BioMed Central and take full advantage of:}

- Convenient online submission

- Thorough peer review

- No space constraints or color figure charges

- Immediate publication on acceptance

- Inclusion in PubMed, CAS, Scopus and Google Scholar

- Research which is freely available for redistribution

Submit your manuscript at www.biomedcentral.com/submit 